\title{
Expression and Purification of Recombinant Superoxide Dismutase (PaSOD) from Psychromonas arctica in Escherichia coli
}

\author{
Jumee Na, Hana Im, ${ }^{\dagger}$ and Kyunghee Lee* \\ Department of Chemistry and ${ }^{\dagger}$ Department of Molecular Biology, Sejong University, Seoul 143-747, Korea \\ *E-mail: khlee@sejong.ac.kr
}

Received May 23, 2011, Accepted June 2, 2011

\begin{abstract}
The psychrophilic bacteria Psychromonas arctica survives at subzero temperatures by having adapted several protective mechanisms against freezing and oxidative stresses. Many reactive oxygen species are likely generated in $P$. arctica as a result of reduced metabolic turnover rates. A previous study identified the pasod gene for superoxide dismutase from $P$. arctica using a series of PCR amplifications. Here, upon cloning into a His-tag fused plasmid, the sod gene from P. arctica (pasod) was successfully expressed by IPTG induction. His-tagged PaSOD was subsequently purified by $\mathrm{Ni}^{2+}$-NTA affinity chromatography. The purified PaSOD exhibited a higher SOD activity than that of Escherichia coli (EcSOD) at all temperatures. The difference in activity between PaSOD and EcSOD becomes even more significant at $4{ }^{\circ} \mathrm{C}$, indicating that PaSOD plays a functional role in the cold adaptation of $P$. arctica in the Arctic.
\end{abstract}

Key Words : Psychrophilic, Superoxide dismutase, Cold-adaptation, Chaperone

\section{Introduction}

Psychrophilic bacteria have adapted to survive in a permanently cold environment such as the Arctic by optimizing enzymes and cellular membranes. ${ }^{1}$ The full molecular mechanisms by which psychrophilic bacteria have acquired their abilities to survive in freezing temperatures remain uncertain, although comparisons of psychrophilic and mesophilic bacteria have provided clues about cold adaptation. ${ }^{2}$ In $P$. arctica, several chaperone proteins have been investigated to elucidate the cold resistance of psychrophilic bacteria in polar environments. For example, cold shock proteins (Csps) known as RNA chaperones ${ }^{3}$ were induced by temperature changes down to $10^{\circ} \mathrm{C} .{ }^{4}$ The Csp genes encoding $\mathrm{CspA}_{\mathrm{pa}}$ were identified using conserved sequences exhibiting 56\% homology to E. coli CspA. When expressed in $E$. coli, these proteins dramatically increased the host's cold resistance by more than tenfold. The tig gene encoding the trigger factor PaTF, which exhibits a peptidyl-propyl cistrans isomerase (PPIase) activity, was also identified in $P$. arctica and cloned to provide His tags for purification. ${ }^{5}$ Biochemical analyses showed that the expressed PaTF was functional in PPIase assays and in in vivo cold-resistance assays. Studies on DnaK and ClpB from P. arctica (PaDnaK and $\mathrm{PcClpB}$ ) have provided further examples of chaperone proteins that play various roles in cold adaptation. ${ }^{6}$

Since the ozone layer over the Arctic has thinned due to colder than normal temperatures, psychrophilic bacteria have become more exposed to high levels of ultraviolet

Abbreviations: SOD; superoxide dismutase, PCR; polymerase chain reaction, IPTG; isopropyl- $\beta$-D-1-thiogalactopyranoside, NBT; nitro blue tetrazolium, WST-1; 2-(4-iodophenyl)-3-(4-nitrophenyl)-5-(2,4disulfophenyl)-2H-tetrazolium radiation, ${ }^{7}$ leading to increased oxidative stresses. In addition, metabolic rates are decreased at freezing temperatures, which leads to an accumulation of excess electrons in the respiratory chains, generating numerous reactive oxygen species (ROS). ${ }^{8}$ To prevent damage from ROS, aerobic cells have adopted sophisticated defense mechanisms employing antioxidant enzymes such as superoxide dismutase (SOD) and catalase (CAT). ${ }^{9}$

SOD is a ubiquitous enzyme that detoxifies ROS by catalyzing the dismutation of the superoxide anion into $\mathrm{O}_{2}$ and $\mathrm{H}_{2} \mathrm{O}_{2} \cdot{ }^{10}$ Four distinct SODs are expressed in bacteria (FeSOD, MnSOD, CuZnSOD, and NiSOD), differing by the type of binding metal cofactors. FeSOD and MnSOD are structurally similar and are composed of an N-terminal domain with a long $\alpha$-antiparallel hairpin and a C-terminal $\alpha+\beta$ domain with three-stranded antiparallel $\beta$-sheets. ${ }^{11}$ CuZnSODs with $\beta$-barrels ${ }^{12}$ and NiSODs with four-helix bundles have been also reported. ${ }^{13}$

Defining which type of metal cofactor these SODs employ is difficult based solely on sequence homology and structural information. However, depending on the metal specificity, their oligomeric properties and selective inhibition properties can be differentiated. In solution, FeSODs form homodimers, ${ }^{14}$ while MnSODs form homotetramers. ${ }^{15}$ Regarding inhibition, FeSOD is inactivated by $\mathrm{H}_{2} \mathrm{O}_{2}$ not by cyanide, while MnSOD is not influenced by either but by azide. $^{16}$

Thus far, three psychrophilic SODs have been isolated, including cold-active SODs from Pseudoalteromonas haloplanktis, Marinomonas sp. NJ522 and Psychromonas arctica. ${ }^{17-19}$ Recently, the first crystal structure of FeSOD from the cold-adapted and fish-pathogenic bacterium Aliivibrio salmonicida (AsFeSOD) was determined. ${ }^{20}$ Our group has previously reported the existence of SOD in $P$. arctica 
(PaSOD), whose genomic sequence has not yet been elucidated. ${ }^{18}$ We were able to identify the coding gene for sod from $P$. arctica using a combination of gradient PCR with homologous primers and LA PCR in vitro cloning technology. Overexpression of PaSOD in Escherichia coli and the purification of recombinant PaSOD were carried out to examine the functional role of PaSOD in cold adaptation. The current study describes the development of an expression system for PaSOD under IPTG induction, purification of PaSOD by affinity chromatography, and biochemical characterization of PaSOD relative to EcSOD.

\section{Experimental Section}

Bacterial Stains and Chemicals. KOPRI22215 (donated by KOPRI, Korea Polar Research Institute) was identified as Psychromonas arctica from its 16S rDNA gene sequence (personal communication). P. arctica was cultured at $15^{\circ} \mathrm{C}$ in Difco ${ }^{\mathrm{TM}}$ marine broth medium (Difco, Grand Island, NY). Cell growth of BL21(DE3) transformants grown in LB medium (trypon $10 \mathrm{~g}$, yeast extract $5 \mathrm{~g}, \mathrm{NaCl} 10 \mathrm{~g}$ per liter) with appropriate antibiotics was monitored by measuring optical densities at $600 \mathrm{~nm}\left(\mathrm{OD}_{600 \mathrm{~nm}}\right)$. The total genomic DNA was extracted from collected cells using a Cell DNA isolation kit (GeneAll, Seoul, Korea). IPTG (isopropyl $\beta$-D1-thiogalactopyranoside) and NBT (nitro blue tetrazolium) were obtained from Sigma-Aldrich (St. Louis, MO). Amicon filters were acquired from Millipore (Billerica, MA) and $\mathrm{Ni}^{2+}$-NTA chelating agarose CL-6B was purchased from Peptron (Daejeon, Korea).

Construction and Expression of PaSOD and EcSOD Plasmids. The pasod gene was amplified in its entirety using the corresponding primers containing Nde1 and BamH1 sites at each end [forward F1: 5'-CAT ATG GCT TTT GAA TTA CCA GCA TTA CC-3', reverse R1: 5'-GGA TCC CTA TAG GGA GAC TGC AGC AAA-3']. After cloning into a pBluescript II vector (Stratagene, La Jolla, $\mathrm{CA}$ ), the NdeI- and BamH1-digested fragments were ligated into the pET28a vector, which had been previously cut using the same enzymes, to provide PaSOD/pET28a. To overexpress PaSOD in E. coli, BL21(DE3) transformed with $\mathrm{PaSOD} / \mathrm{pET} 28 \mathrm{a}$ was cultured at $28^{\circ} \mathrm{C}$ in $\mathrm{LB}^{\mathrm{kan}}$ to an $\mathrm{OD}_{600 \mathrm{~nm}}$ of 0.5 , at which point IPTG was added to a final concentration of $0.5 \mathrm{mM}$. After a 5 -h incubation with IPTG, cells were harvested and frozen at $-70{ }^{\circ} \mathrm{C}$.

Cloning and expression of the corresponding E. coli SODs were performed similarly. Genomic DNA was purified by a Cell DNA isolation kit (Exgene Cell SV, GeneAll) from E. coli strain DH5 that had been cultured overnight in LB media. The sod gene from $E$. coli genomic DNA was amplified via PCR using F2 and R2 primers for Fe-type SOD (EcFeSOD) and $\mathrm{F} 3$ and R3 primers for Mn-type EcSOD (EcMnSOD). The sequences of primers were as follows: F2, 5'-AGG AGA GCT AGC ATG TCA TTC GAA TTA-3'; R2, 5'-CAT TTG GGA TCC GTT ATT ATG CAG CGA-3'; F3, 5'-CTG GAG ATG CAT ATG AGC TAT ACC CTG-3'; and R3, 5'-GCC TCA TTG CAG CAG GAT CCA AAT GAT-3'.
The annealing temperatures were $58{ }^{\circ} \mathrm{C}$ and $56{ }^{\circ} \mathrm{C}$, respectively.

Purification of PaSOD by $\mathrm{Ni}^{2+}$-Affinity Chromatography. Bacterial cells were lysed on ice by incubating with binding buffer [50 mM Tris-Cl (pH 7.9), $250 \mathrm{mM} \mathrm{NaCl}, 8$ $\mathrm{mM}$ imidazole] for $30 \mathrm{~min}$. After sonication, protein extracts were collected by centrifugation at $4{ }^{\circ} \mathrm{C}$ for $20 \mathrm{~min}$, separated by $10 \%$ SDS-PAGE, and examined by Coomassie blue staining. His-tagged PaSOD induced by IPTG was further purified by $\mathrm{Ni}^{2+}$-NTA chromatography. After loading a protein extract sample, the column was washed with ten volumes of binding buffer [50 mM Tris-Cl (pH 7.9), 250 $\mathrm{mM} \mathrm{NaCl}, 8 \mathrm{mM}$ imidazole), six volumes of washing buffer [50 mM Tris-Cl (pH 7.9), $250 \mathrm{mM} \mathrm{NaCl}, 20 \mathrm{mM}$ imidazole), and finally six volumes of elution buffer $[50 \mathrm{mM}$ Tris$\mathrm{Cl}$ (pH 7.9), $250 \mathrm{mM} \mathrm{NaCl}, 800 \mathrm{mM}$ imidazole). The overexpressed PaSOD was obtained with successive buffer exchange using Amicon-10 filtration (Millipore). The concentration of PaSOD was measured by the Bradford method using bovine serum albumin as a standard ${ }^{21}$ and the purity of PaSOD was confirmed by $10 \%$ SDS-PAGE.

Identification of SOD Activity on Native PAGE Gels. The purified SODs were separated on native polyacrylamide gels (PAGE) for SOD activity staining as follows. ${ }^{22}$ Native gels without SDS and $\beta$-mercaptoethanol were prepared with $10 \%$ polyacrylamide and run at $120 \mathrm{~V}$ for $1 \mathrm{~h}$. The native gels were incubated in $1.23 \mathrm{mM}$ NBT for $15 \mathrm{~min}$ followed by incubation in $100 \mathrm{mM}$ phosphate buffer $(\mathrm{pH}$ 7.0) containing $0.028 \mathrm{mM}$ riboflavin and $28 \mathrm{mM}$ TEMED with gentle shaking at $75 \mathrm{rpm}$ in the dark. After brief washing, the gels were illuminated with a fluorescent lamp for $15 \mathrm{~min}$.

The Specific SOD Activity of Expressed SODs. The specific activity of superoxide dismutase was measured spectroscopically with a SOD assay kit with WST (Dojindo Molecular Technologies, Inc., Tokyo, Japan). In principle, the reduction of WST-1 (2-(4-iodophenyl)-3-(4-nitrophenyl)-5-(2,4-disulfophenyl)-2H-tetrazolium) inhibition by the superoxide anion is correlated with the inhibition of xanthine oxidase by the SOD enzyme. ${ }^{9}$ The $\mathrm{IC}_{50}$ value, which indicates the concentration of SOD at which $50 \%$ inhibition activity was observed, was determined according to the expression below by measuring the OD of the solution at $450 \mathrm{~nm}$ in a 96-well plate following a 20-min incubation at $37^{\circ} \mathrm{C}$. One unit of SOD is defined as the amount of the enzyme in $20 \mu \mathrm{L}$ sample solution at $\mathrm{IC}_{50}$. The SOD activity was calculated using the following equation.

$$
\begin{aligned}
& \text { SOD activity (inhibition rate } \%) \\
& \qquad=\frac{\left(A_{\text {blank } 1}-A_{\text {blank } 3}\right)-\left(A_{\text {sample }}-A_{\text {blank } 2}\right)}{\left(A_{\text {blank } 1}-A_{\text {blank } 3}\right)} \times 100
\end{aligned}
$$

\section{Results and Discussions}

Cloning and Purification of PaSOD and EcSOD. The pasod gene is 594 bp in length ${ }^{18}$ and expected to encode 


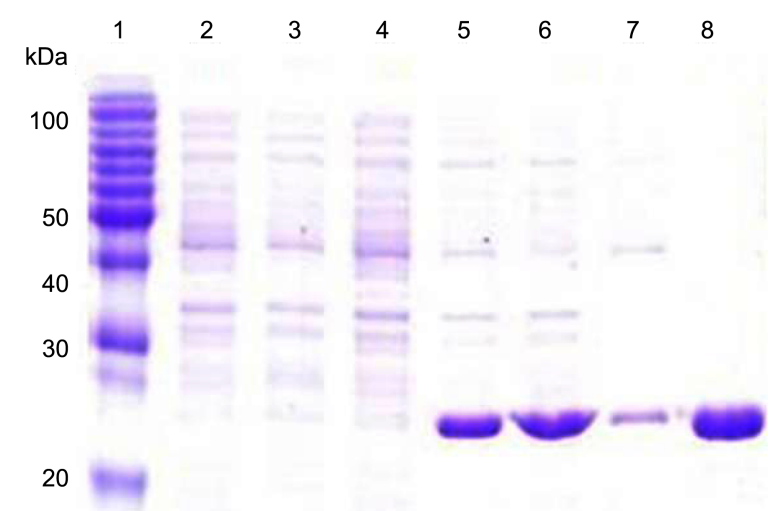

Figure 1. SDS-PAGE gel analysis to monitor the induction and purification of PaSOD. BL21(DE3) cells transformed with pET28a (lanes 2 and 3) and pasod/pET28a (lanes 4 and 5) were cultured in the absence (lanes 2 and 4) or presence of $0.5 \mathrm{mM}$ IPTG (lanes 3 and 5). Each sample was removed from a 1-mL culture separated on $12 \%$ SDS-PAGE and stained with Coomassie blue. Then 250 $\mathrm{mL}$ of bacterial cell culture was treated with $0.5 \mathrm{mM}$ IPTG, lysed, and separated into pellet (lane 6) and supernatant fractions (lane 7). The supernatant fractions were loaded onto $a \mathrm{Ni}^{2+}-\mathrm{NTA}$ resin and eluted with $800 \mathrm{mM}$ imidazole. After Amicon filtration, the purity of PaSOD was examined by SDS-PAGE (lane 8). Size markers for proteins were separated along with the samples in lane 1.

PaSOD with 197 amino acids. To purify the expressed PaSOD in a one-step process using affinity chromatography, we developed a His-tagging system using the pET28a vector for pasod cloning. After amplification using two primers containing Ndel and BamH1 restriction sites, the pasod gene was successfully cloned into the pET28a vector, yielding PaSOD/pET28a. Overexpression of PaSOD in BL21(DE3) was carried out in the presence of $0.5 \mathrm{mM}$ IPTG and examined by $10 \%$ SDS-PAGE and Coomassie blue staining (Fig. 1). Most expressed PaSOD proteins remained in the pellet forming inclusion body. We have tried to resolubilize the protein by on-column refolding but failed. The soluble fraction of His-tagged PaSOD (lane 7 in Fig. 1) was further purified by NTA-chromatography to provide pure PaSOD (lane 8 in Fig. 1).

Cloning of $E$. coli SOD in pET28a was also carried out by PCR amplification of the E. coli genomic DNA with the appropriate primers designed in this study. Induction of EcMnSOD and EcFeSOD by IPTG was confirmed by $10 \%$ SDS-PAGE analysis (Fig. 2). The molecular mass of each enzyme was estimated as $23 \mathrm{kDa}$ for EcMnSOD (206 aa) and $21 \mathrm{kDa}$ for EcFeSOD (193 aa).

The Specific SOD Activity of Expressed SODs. The specific activity of SOD was measured spectroscopically using a xanthine oxidase system (Fig. 3(a)). Even if this method is indirect one including another enzyme, xanthine oxidase, it gave us reliable results with high sensitivity. The effect of temperature on PaSOD activity could be evaluated by comparing with positive control, in our case, EcSODs. To monitor the activity of SODs, $\mathrm{IC}_{50}$ values were determined by measuring the solution absorbance at $450 \mathrm{~nm} .{ }^{9}$ Note that PaSOD exhibited the highest activity of the enzymes tested

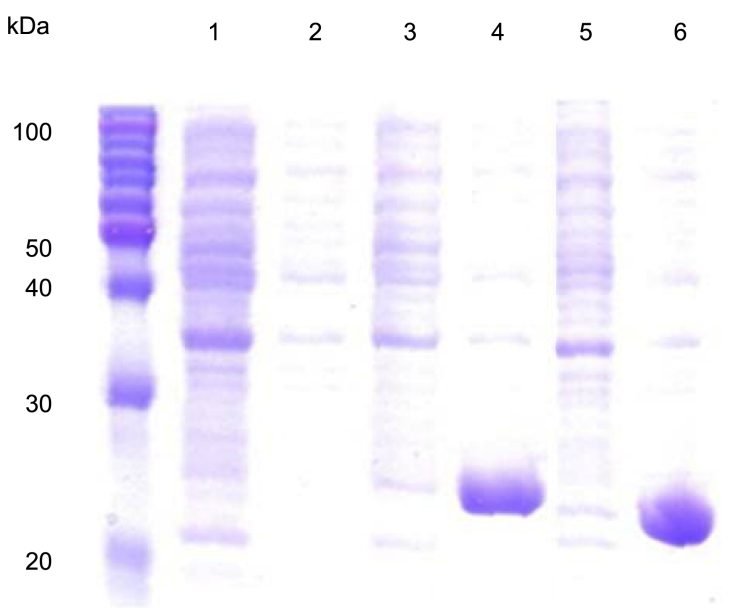

Figure 2. Expression of Fe- and Mn- type EcSODs. Mn-type Ecsod (lanes 3 and 4) and Fe-type Ecsod genes (lanes 5 and 6) were cloned in pET28a and expressed for $3 \mathrm{~h}$ in BL21(DE3) with (lanes 2, 4, and 6) or without $0.3 \mathrm{mM}$ IPTG induction (lanes 1, 3, and 5). Control experiments were performed with the pET28a vector alone (lanes 1 and 2).

(Fig. 3(b)). The $\mathrm{IC}_{50}$ of PaSOD $(0.35 \mu \mathrm{g} / \mathrm{mL})$ was slightly lower than that of Ec-FeSOD $(0.49 \mu \mathrm{g} / \mathrm{mL})$. EcMnSOD $\left(\mathrm{IC}_{50}\right.$ of $\left.2.68 \mu \mathrm{g} / \mathrm{mL}\right)$ exhibited a drastically lower activity than that of PaSOD by a factor of seven. To assess the role of PaSOD in cold adaptation, SOD-specific activities were measured as a function of decreasing temperature from $37{ }^{\circ} \mathrm{C}$ to $4{ }^{\circ} \mathrm{C}$ (Fig. 3(c)). It should be noted that PaSOD showed higher activities than those of EcFeSOD at all temperatures. PaSOD had the highest SOD activities at $4{ }^{\circ} \mathrm{C}$ as we expected. However, enhancement of the SOD activity upon decreasing temperature was also found even in mesophilic EcSOD, probably due to the high solubility of oxygen at low temperature. Nonetheless, the difference in activity between PaSOD and EcSOD becomes even more noteworthy at $4{ }^{\circ} \mathrm{C}$, indicating that PaSOD plays a functional role in the cold adaptation of $P$. arctica in the Arctic.

In-gel Assay for Specific Inhibition of SOD. In order to check the SOD activity in native gel, purified SOD proteins were separated on native PAGE and stained with NBT. We observed that PaSOD displayed a higher SOD activity than EcFeSOD, as demonstrated by twofold serial dilutions (Fig. 4(a)). PaSOD exhibited the SOD activity at the lowest level of $0.063 \mathrm{mg}$. The inhibition patterns exhibited by several inhibitors can be rather specific depending on the metal cofactor incorporated in the SOD. For example, FeSOD is inhibited by $\mathrm{H}_{2} \mathrm{O}_{2}$, but not by cyanide. $\mathrm{Cu} / \mathrm{ZnSOD}$ can be blocked by both of these compounds while MnSOD is unaffected in the presence of either one. The metal-specific inhibition of PaSOD was characterized using the native gel assay with EcFeSOD as a control (Fig. 4(b)). PaSOD showed a very similar inhibition pattern as that of EcFeSOD and was blocked by $\mathrm{H}_{2} \mathrm{O}_{2}$ but not by $\mathrm{CN}^{-}$. This result implies that PaSOD behaves as an Fe-type SOD, which is in a good agreement with homology comparisons of its amino acid sequence. 
(a)

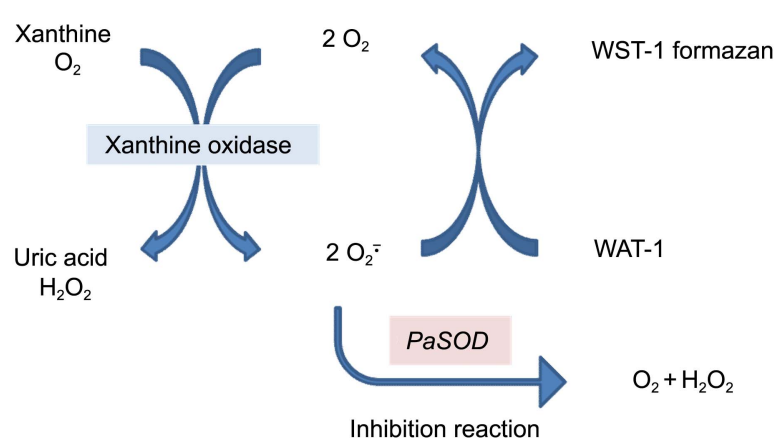

(b)

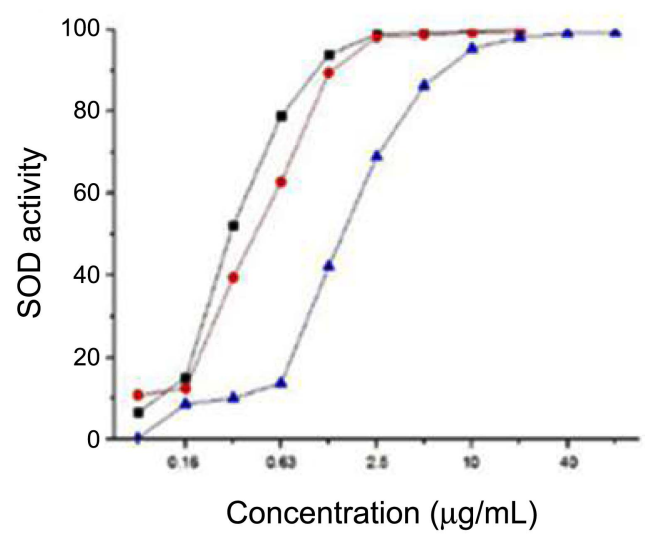

(c)

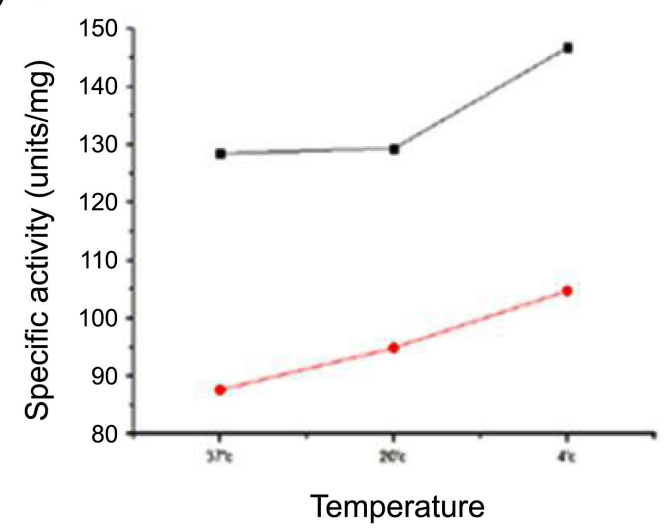

Figure 3. Activity of xanthine oxidase inhibited by SODs. (a) The specific activity of SOD was measured spectroscopically with a SOD assay kit-WST in which the reduction of WST-1 was inhibited by the superoxide anion. (b) IC $_{50}$ values [PaSOD (square), EcFeSOD (circle), and EcMnSOD (triangle)] were determined by measuring the absorbance of solution at $450 \mathrm{~nm}$. (c) Specific activities (units//mg) were measured after incubation at $37{ }^{\circ} \mathrm{C}, 20^{\circ} \mathrm{C}$, and $4{ }^{\circ} \mathrm{C}$ for PaSOD (squares) and EcFeSOD (circles).

(a)

EcFeSOD

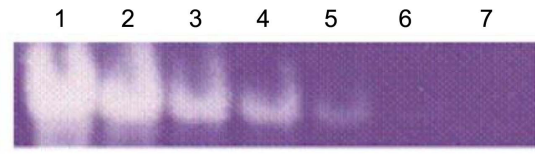

PaSOD

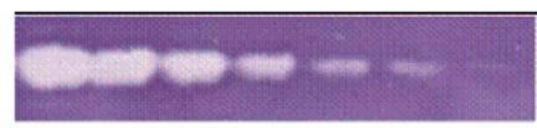

(b)

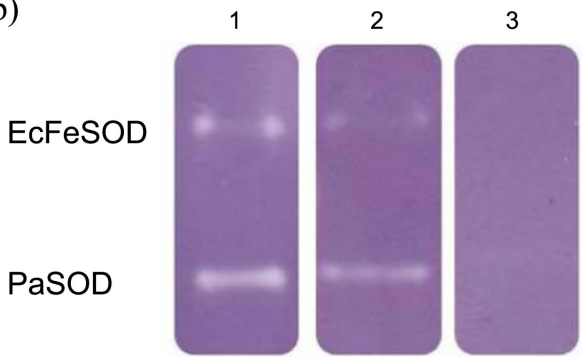

Figure 4. NBT staining of SOD activities on native gel. (A) The SOD activities of purified EcFeSOD and PaSOD were examined on native gel by a two-fold serial dilution followed by NBT staining. The amount of SOD used were $4 \mu \mathrm{g}$ (lane 1), $2 \mu \mathrm{g}$ (lane 2), $1 \mu \mathrm{g}$ (lane 3), $0.5 \mu \mathrm{g}$ (lane 4), $0.25 \mu \mathrm{g}$ (lane 5), $0.13 \mu \mathrm{g}$ (lane 6), and $0.063 \mu \mathrm{g}$ (lane 7). (b) Blocking of EcFeSOD and PaSOD activity by $\mathrm{CN}^{-}$(lane 2) or $\mathrm{H}_{2} \mathrm{O}_{2}$ (lane 3) was also monitored. Each SOD protein without inhibitor treatment was compared to the control (lane 1).
Although structural data for PaSOD are lacking, the SOD from Psychromonas ingrahamii (PiSOD) was categorized as a Fe/Mn SOD with an $\alpha$-hairpin $\mathrm{N}$-terminal and $\mathrm{C}$-terminal $\alpha+\beta$ domains with three-stranded antiparallel sheets. ${ }^{23}$ Since PaSOD (197 aa) from P. arctica displays significant homology with PiSOD (193 aa) by a score of 83 in ClustalW2 analyses (EBI server), ${ }^{24}$ PaSOD likely contains similar protein domains. ClustalW2 analyses have also indicated that PaSOD is more homologous with EcFeSOD, with a score of 75, than with EcMnSOD, with a score of 42. This comparison suggests that PaSOD is a Fe-type SOD. SOD inhibition assays focusing on the metal dependence of SOD activity also showed that PaSOD was sensitive to $\mathrm{H}_{2} \mathrm{O}_{2}$ inhibition, a further characteristic of Fe-type SODs. Further studies employing atomic absorption spectroscopy may be necessary to confirm the metal content in PaSOD. ${ }^{25}$ The search for a Mn-type PaSOD by PCR amplification with the proper primers has been unsuccessful. At this point, one cannot rule out that Mn- or Ni-type PaSODs may be key factors in the cold-temperature survival of $P$. arctica. Further exploration of other type of sod genes from P. arctica is in progress.

Acknowledgments. We wish to appreciate KOPRI (Korea Polar Research Institute) for providing the psychrophilic bacterial strain. This work was supported by a grant (R01- 
2006-000-11154-0) from the Basic Research Program of the Korea Science and Engineering Foundation.

\section{References}

1. 1. Gerday, C.; Aittaleb, M.; Arpigny, J.L.; Baise, E.; Chessa, J.P.; Garsoux, G.; Petrescu, I.; Feller, G. Biochim. Biophys. Acta 1997, $1342,119$.

2. Feller, G.; Gerday, C. Cell Mol. Life Sci. 1997, 53, 830.

3. Jiang, W.; Hou, Y.; Inouye, M. J. Biol. Chem. 1997, 272, 196.

4. Jung, Y. H.; Yi, J. Y.; Jung, H. J.; Lee, Y. K.; Lee, H.K.; Naicker, M. C.; Uh, J. H.; Jo, I. S.; Jung, E. J.; Im, H. Protein J. 2010, 29, 136.

5. Lee, K.; Choi, H.; Im, H. Curr. Microbiol. 2009, 59, 160.

6. Choi, A.; Na, J.; Sung, M.; Im, H.; Lee, K. Bull. Korean Chem. Soc. 2010, 31, 887.

7. Coldiron, B. M. J. Am. Acad. Dermatol. 1992, 27, 653.

8. Selman, C.; McLaren, J. S.; Himanka, M. J.; Speakman, J. R. Free Radic. Biol. 2000, 28, 1279.

9. Matés, J. M.; Sánchez-Jiménez, F. Front Biosci 1999, 4, D339.

10. Bannister, J. V.; Bannister, W. H.; Rotilio, G. CRC Crit. Rev. Biochem. 1987, 22, 111

11. Knapp, S.; Kardinahl, S.; Hellgren, N.; Tibbelin, G.; Schafer, G.; Ladenstein, R. J. Mol. Biol. 1999, 285, 689.

12. Tainer, J. A.; Getzoff, E. D.; Beem, K. M.; Richardson, J. S.;
Richardson, D. C. J. Mol. Biol. 1982, 160, 181.

13. Barondeau, D. P.; Kassmann, C. J.; Bruns, C. K.; Tainer, J. A.; Getzoff, E. D. Biochemistry 2004, 43, 8038.

14. Yikilmaz, E.; Porta, J.; Grove, L. E.; Vahedi-Faridi, A.; Bronshteyn, Y.; Brunold, T. C.; Borgstahl, G. E.; Miller, A. F. J. Am. Chem. Soc. 2007, 129, 9927.

15. Takeda, Y.; Avila, H. Nucleic. Acid Res. 1986, 14, 4577.

16. Fournier, M.; Zhang, Y.; Wildschut, J. D.; Dolla, A.; Voordouw, J. K.; Schriemer, D. C.; Voordouw, G. J. Bacteriol. 2003, 185, 71.

17. Castellano, I.; Di Maro, A.; Ruocco, M. R.; Chambery, A.; Parente, A.; Di Martino, M. T.; Parlato, G.; Masullo, M.; De Vendittis, E. Biochimie 2006, 88, 1377.

18. Na, J.; Lee, K. Bull. Korean Chem. Soc. 2010, 31, 2410.

19. Zheng, Z.; Jiang, Y. H.; Miao, J. L.; Wang, Q. F.; Zhang, B. T.; Li, G. Y. Biotechnol Lett. 2006, 28, 85.

20. Pedersen, H. L.; Willassen, N. P.; Leiros, I. Acta Crystallogr. Sect. F Struct. Biol. Cryst. Commun. 2009, 65, 84.

21. Bradford, M. M. Anal. Biochem. 1976, 72, 248.

22. Beauchamp, C.; Fridovich, I. Anal. Biochem. 1971, 44, 276.

23. Sonnhammer, E. L.; Eddy, S. R.; Durbin, R. Proteins 1997, 28, 405.

24. Thompson, J. D.; Higgins, D. G.; Gibson, T. J. Nucleic. Acids Res. 1994, 22, 4673

25. Gud, F. X.; Shi-Jin, E.; Liu, S. A.; Chen, J.; Li, D. C. Mycoloqia 2008, 100, 375 . 